\title{
TRANSIENT BEAM-LOADING DETECTION IN AN SNS CAVITY- SIMULATIONS AND INITIAL MEASUREMENTS*
}

\author{
Y. Zhang, I. E. Campisi, C. Deibele, J. Galambos, \\ S. D. Henderson, H. Ma, Y. Kang, J. Wilson \\ Spallation Neutron Source, ORNL, Oak Ridge, TN 37831, USA
}

\section{Abstract}

Beam phase measurement based on the detection of a transient beam-loading signal in a SC cavity has the potential to become a very fast linac tune-up technique, especially for an electron linac, as it may precisely set up the cavity's synchronous phase. A large signal-to-noise ratio is critical in the development of a transient detector technique. This paper discusses the study of a transient detector in the Spallation Neutron Source proton linac and the major challenge for this method: stochastic noise in the rf system, which reduces the precision and increases the time needed for accurate phase measurement. Using simulation studies with a beam-cavity model, signal measurement with the Low-Level RF system, and the experiment with prototype detectors, we analyzed the influence of noise on phase measurement.

\section{INTRODUCTION}

The transient beam-loading signal in an SC cavity for high-intensity pulsed beams is utilized to measure beam phase in an electron linac at DESY [1] because the beam signal phase is exactly $180^{\circ}$ to the RF crest. It provides an additional synchronous phase measurement for SC cavities based on beam-cavity interaction techniques. Two other methods are (1) introducing RF modulations into an SC cavity for phase measurement [2], which is limited to $\mathrm{CW}$ beams or long-pulse operations, and (2) measurement of drifting beams, which is applied to short pulse beams. The cavity needs to be turned off and on during the drifting beam measurement [3], so it is time consuming. A precise and fast linac tune-up technique is needed for an SC linac with multiple independently phased cavities. A transient detector has such potential, but it has to properly handle the stochastic noise in the RF systems.

\section{TRANSIENT DETECTION}

In the transient detector developed at DESY, a 100-ns delay is utilized to subtract the carrier frequency from the signal and to measure the tiny transient of a single beam bunch [1]. To reach a similar signal level, we need a 250 ns delay for accumulation of no less than 100 beam bunches because the single bunch transient in an SNS cavity is 100 times less than that of DESY. Table 1 lists some of the parameters of the two systems. A transient detector with a longer delay is more prone to errors caused by temperature variations and imperfections of the cavity RF loop. We used a 150-ns delay as a compromise for the signal level to reduce sensitivity to errors.

* SNS is managed by UT-Battelle, LLC, under contract DE-AC0500OR22725 for the U.S. Department of Energy.
Table 1: Some Major Parameters of the Two Systems

\begin{tabular}{|l|cc|}
\hline & DESY & SNS \\
\hline Loaded Q & $4 \times 10^{6}$ & $7 \times 10^{5}$ \\
$\mathrm{R} / \mathrm{Q}$ & $1.0 \mathrm{k} \Omega$ & $483.6 \Omega$ \\
Frequency & $1.3 \mathrm{GHz}$ & $805 \mathrm{MHz}$ \\
Total charge in one bunch & $1 \mathrm{nC}$ & $0.1 \mathrm{nC}$ \\
Design $\beta$ & 1.0 & 0.81 \\
\hline
\end{tabular}

One proposed SNS transient detector is shown in Fig. 1. The major difference between it and DESY's detector is a $150-$ ns delay instead of a 100-ns delay.

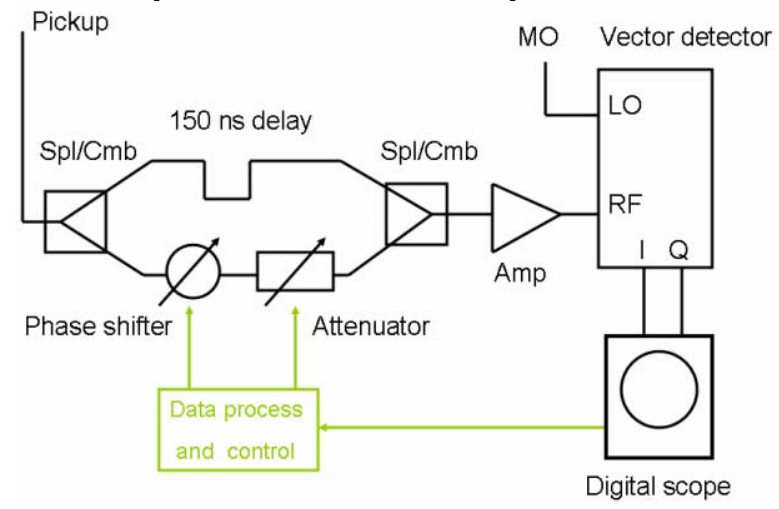

Figure 1: One of the proposed SNS transient detectors.

\section{SIMULATION}

A beam-cavity model was developed to simulate beambunch-induced transients in an SNS cavity. The model uses the cavity-equivalent parallel RLC circuit method; the cavity voltage is the sum of the RF generator voltage, $V_{r f}$, and the beam-loading-induced voltage, $V_{L}$. In the drifting-beam measurement, the model shows close agreement with other methods [3], such as phase scan [4]. When a beam bunch train is transported through a cavity, particles also experience a self-induced voltage, $V_{b 0}$ :

$$
\begin{aligned}
& V=V_{r f} \cdot \cos \varphi_{r f}-V_{L}-\frac{1}{2} V_{b 0} \\
& V_{L}=V_{b 0} \cdot\left\{\exp \left[-T_{b}\left(\frac{1}{T_{F}}+j \cdot d \omega\right)\right]+\exp \left[-2 \cdot T_{b}\left(\frac{1}{T_{F}}+j \cdot d \omega\right)\right]+\ldots\right\} \\
& V_{b 0}=I(\omega) \cdot R_{s h} \cdot \frac{T_{b}}{T_{F}}
\end{aligned}
$$

where $\varphi_{\mathrm{rf}}$ is the RF phase, $T_{\mathrm{b}}$ is the beam bunch period, $T_{\mathrm{f}}$ is the filling time of the cavity, $R_{\mathrm{sh}}$ is the shunt impedance, $I$ is the image current of the beam bunch in the frequency domain, $\omega$ is the angular frequency, and $d \omega$ is the cavity detuning.

Because the transient signal generated by a single beam bunch in the SNS cavity is so small compared with that of DESY, our first question was whether we could measure 
it at SNS reliably. Figure 2 shows the simulations of a $750-\mathrm{MeV}, 38-\mathrm{mA}$ proton-beam-generated transient in a high-beta cavity for a pulse length of up to $0.5 \mu \mathrm{s}$.

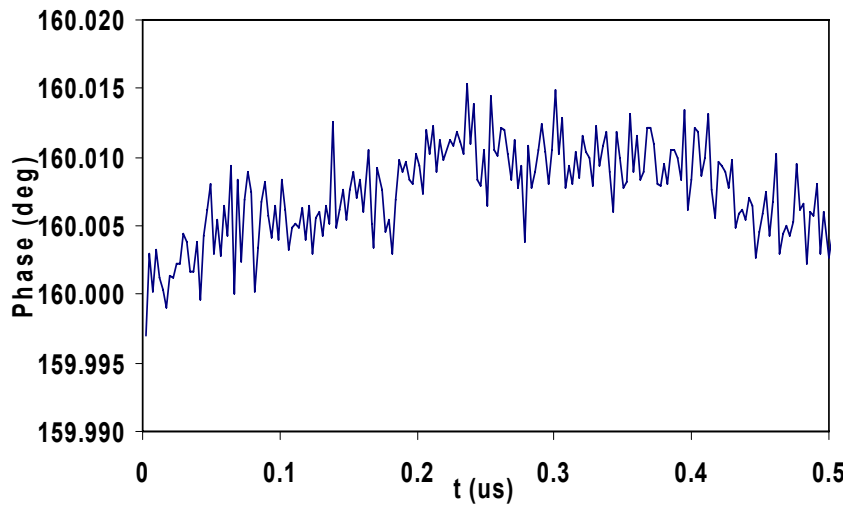

Figure 2: Cavity phase under transient beam loadings.

In the simulation, the cavity field amplitude was 15 $\mathrm{MV} / \mathrm{m}$, and the noise amplitude was approximately $1 \times$ $10^{-4}$ of the RF signal. The simulation result shows that a precise phase measurement with a single beam bunch is impossible at SNS, but accumulation of 100- to 200-ns beam bunch signals can be utilized to measure the beam phase. Since all of the six TM010 passband modes are included, we may also see a frequency close to $0.8-\mathrm{MHz}$ which mainly comes from the beam-induced $5 / 6 \pi$ mode, if we extend the beam pulse length in the simulation.

Stochastic noise in the cavity RF system is one of the toughest obstacles to a precise phase measurement with the transient detector because the transient signal is only approximately $1 \times 10^{-3}$ of the cavity acceleration gradient. Figure 3 shows the simulated performance of the detector with RF noise of $1 \times 10^{-3}$ in amplitude. Figures 4 and 5 are phase errors during the beam bunch accumulations with RF noise of $1 \times 10^{-3}$ and $1 \times 10^{-4}$, respectively. RF modulations from the feedback control loop are omitted in all of these simulations.

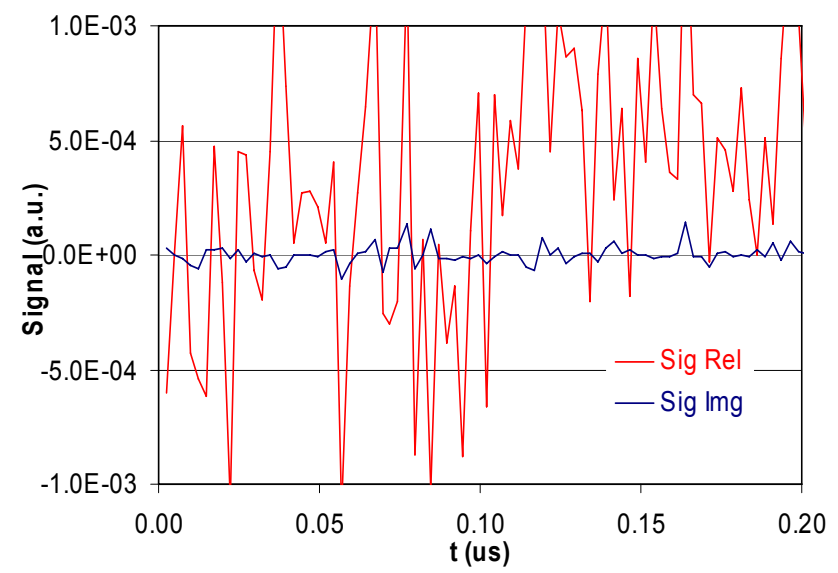

Figure 3: Beam accumulations with a $1 \times 10^{-3} \mathrm{RF}$ noise.

From the simulation results, we see that it is possible to measure the beam transient in the SNS cavity with noise of $1 \times 10^{-3}$ the RF signal, but phase errors of several tens of degrees are expected for a measurement with only one beam pulse although it may consist of up to a few hundred bunches. To measure the cavity phase accurately (within a few degrees) with only one beam pulse which could be necessary for a fast tune-up algorithm for the SC linac, noise in the cavity RF system and in the transient detector should be below $1 \times 10^{-4}$ of the RF signal. If it needs a phase within $\pm 1^{\circ}$, one should reduce RF noise in the relevant systems to $1 \times 10^{-5}$. In the short-pulse mode, phase error caused by cavity detuning can be ignored in the measurement, and other passband modes can be dealt with too.

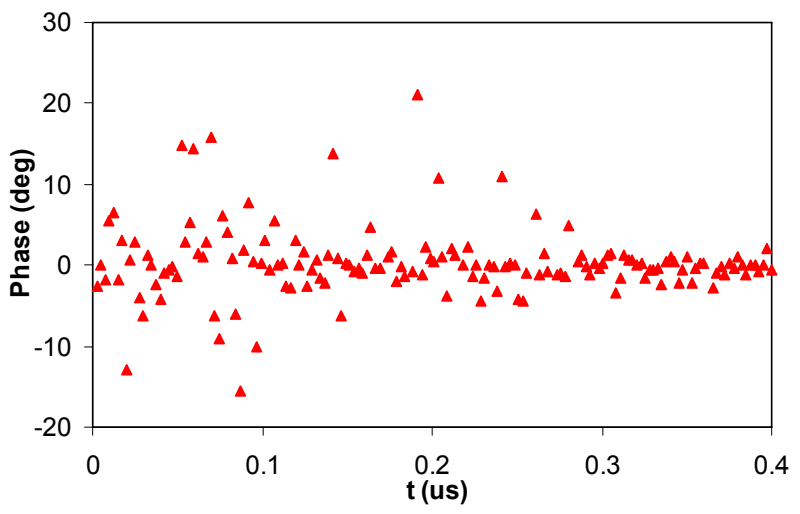

Figure 4: Beam phase errors with a $1 \times 10^{-3} \mathrm{RF}$ noise.

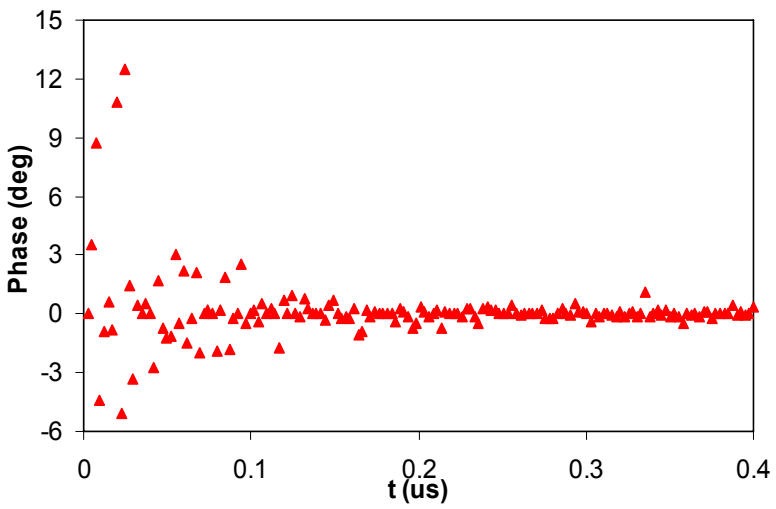

Figure 5: Beam phase errors with a $1 \times 10^{-4} \mathrm{RF}$ noise.

\section{MEASUREMENT}

Beam transients in a high-beta cavity for a $750-\mathrm{MeV}$ beam (peak current $22 \mathrm{~mA}$ ) were measured with the cavity LLRF system. During the measurement, the adaptive feed forward of the LLRF control was turned off to avoid its interference with the transient detection. Because the transient is very small, it could not be directly seen in the LLRF waveform. The necessary subtraction of the carrier signal was handled by the measurement software, in which one may use various delays easily. A measured result is shown in Fig. 6.

Beam current was measured with a beam current monitor (Fig. 6). Also shown is the transient amplitude and phase. Since a smaller delay and sampling rate usually lead to a larger noise compared to the tiny beam transient, the signal is completely faded out. In the measurement, the sampling rate and delay are increased to $1 \mu \mathrm{s}$, which flattens the beam transient signal, RF noise as 
well as all other passband frequencies. Beam transients in amplitude and phase are clearly shown with this longer delay, but the measured cavity phase is approximately $40^{\circ}$ away from the actual set point. It is known from comparisons of the measurements with the simulation and with some other measurements that noise in the RF system is not less than $1 \times 10^{-3}$.

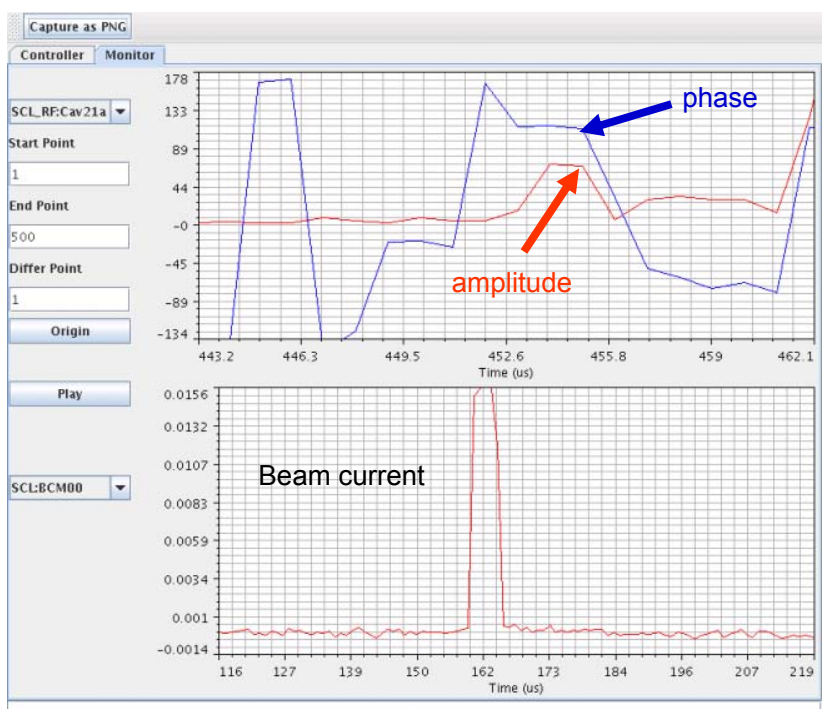

Figure 6: Beam transient measured with the LLRF.

Two prototype detectors were built at SNS in 2006, one 402.5-MHz detector for the normal conducting MEBT rebuncher cavities and one $805-\mathrm{MHz}$ detector for the SC cavities. Bench performance of both detectors was satisfactory. RF noise began showing up, although still at an acceptable level, when the detectors were tested in normal conducting cavities. But RF noise became an impediment to precise phase measurement of beam transients in an SC cavity.

Figure 7 shows one of the transient measurements in an SC cavity for an $840-\mathrm{MeV}, 30-\mathrm{mA}$ peak current proton beam. And the sampling rate is approximately $0.1 \mathrm{~ns}$ in the measurement. The carrier frequency prior to the beam transient is shown in the measured waveform. The fact that the mean of this signal is not zero may be due to the detector not being fully balanced or due to beam chopper leakage. In the measured beam transients, the $0.8-\mathrm{MHz}$ structure is also shown as we explained in the previous simulation studies. The most important information is that the measured signal-to-noise ratio is only approximately two, which is why the measured phase has an error of several tens of degrees.

In the drifting beam study, we learned that a major component of RF noise in the SNS cavities without RF power comes from cross talk with other cavity circuits. Therefore, a bandpass filter may not work efficiently there because they operate at the same frequency, and it is not a mean zero noise in a short pulse [3]. Thus, in the transient measurement, using a smaller sampling interval to average more samples in the same pulse would not effectively reduce the phase error. One solution would be to increase the beam current or the stability of the cavity
RF systems by at least an order of magnitude, although it is not possible with our present resources. Another solution would be to record more beam pulses and average all the signals to reduce the error from noise. At DESY, a hundred beam pulses were used to get phase information that is accurate up to a few degrees by averaging samples [5]. However, it used much of the time needed for phase measurement with the detector, which is also crucial to a fast linac tune-up technique.

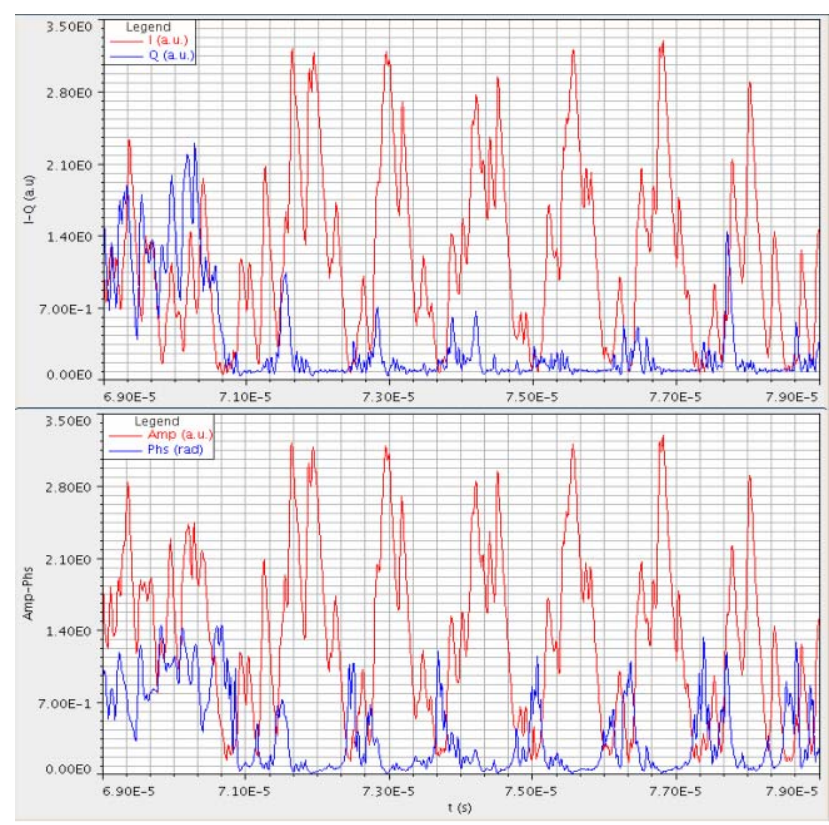

Figure 7: Beam transient measured with a detector. Upper: I-Q vs time, lower: amplitude-phase.

At SNS, we developed two techniques to precisely measure the SC cavity phase within $\pm 1^{\circ}$ : phase scan and drifting beam. Both need only about 20 beam pulses. They are faster and more precise than the prototype transient detectors. Therefore, the transient detector may become very important to the critical sections in XFEL, where the required stability of the cavity RF system is $10^{-4}$ [6]. It still has a long way to go for application in an SC linac such as the SNS, which has an RF stability of only about $10^{-3}$ to $10^{-2}$.

\section{CONCLUSION}

We studied a transient detector at SNS with simulations and with beam signal measurements in an SC cavity. The existing stochastic noise in the cavity RF system becomes a major concern for a fast and precise phase measurement with this technique.

\section{REFERENCES}

[1] P. Pawlik, M. Grecki, S. Simrock, DIPAC 2005, 108.

[2] S. Sharamentov et al., PRST-AB, 6 (2003) 052802.

[3] Y. Zhang et al., Nucl. Inst. Meth. A 571 (2007) 574.

[4] J. Galambos et al., Linac2006, 174.

[5] A. Brandt, private conversations.

[6] A. S. Hofler et al., Linac2004, 180. 